\title{
ORIGINAL RESEARCH \\ Longitudinal Magnetization Transfer Imaging in Mild to Severe Alzheimer Disease
}

S. Ropele

R. Schmidt

C. Enzinger

M. Windisch

N.P. Martinez

F. Fazekas
BACKGROUND AND PURPOSE: MTI has been proposed as a sensitive technique for studying microstructural brain tissue changes in patients with $A D$, but the course of these changes over time is largely unknown. We therefore used a placebo-controlled study of memantine to follow the evolution of tissue damage in AD by means of MTR measurements and investigated how MTR changes were related to brain atrophy and cognition.

MATERIALS AND METHODS: Twenty-eight patients (76.5 \pm 5.8 years) with mild to moderate AD underwent MTI, brain volume measurements, and cognitive testing at baseline and after 6 and 12 months. Nineteen healthy individuals (73.3 \pm 3.2 years) served as controls. MTI was performed with a 2-minute protocol that was optimized for an enhanced MT effect and reduced motion sensitivity. Global and regional MTR measurements served as correlations with brain volumes and the MMSE score.

RESULTS: AD patients had significantly lower global MTR values than controls, and showed a consistent and significant MTR reduction in all regions investigated over a period of 12 months. These MTR changes were paralleled by a brain tissue loss of $2.2 \%$ per year. Associations between MTR and cognition were found for the hippocampus, putamen, and thalamus, and were more pronounced in the left hemisphere.

CONCLUSIONS: MTI in AD allows the assessment of ongoing global and regional brain damage independent of atrophy, and therefore appears to be a valuable marker for disease-related tissue changes.

ABBREVIATIONS: $A D=$ Alzheimer disease; $F M R I B=$ functional MR imaging of the brain; $M M S E=$ Mini-Mental State Examination; MPRAGE = magnetization-prepared rapid acquisition of gradient echo; $\mathrm{MT}$ = magnetization transfer; $\mathrm{MTI}=$ magnetization transfer imaging; $\mathrm{MTR}=$ magnetization transfer ratio; SAR = specific absorption rate
A $\mathrm{D}$ is the most common cause of dementia in the elderly ${ }^{1}$ and is characterized by progressive degenerative changes of the CNS. Accumulation of neurofibrillary tangles and of extracellular deposits of amyloid $\beta$ are considered the pathologic hallmarks of this process. ${ }^{2}$ The initial pathologic changes are likely to start in the medial temporal lobe and, in particular, in the hippocampus. ${ }^{2-4}$ From there the disorder is thought to propagate to the neocortex, including other microscopic changes like gliosis, demyelination, and neuronal loss. ${ }^{5,6} \mathrm{Un}$ fortunately, these histopathologic changes cannot be detected with conventional MR imaging, which leaves advanced brain tissue loss as the only imaging finding in $\mathrm{AD} .^{7,8}$

MTI is a MR imaging method with high sensitivity for microstructural tissue changes. MTI is based on the exchange of magnetization between tissue water and ${ }^{1} \mathrm{H}$ protons bound to macromolecules, which are especially prevalent in lipids and proteins of myelin. ${ }^{9}$ Therefore, MTI is considered a useful tool for measuring myelin content. However, histopathologic studies have also shown good correlation with neuronal attenuation. ${ }^{10}$ Using spectral selective saturation pulses, MTI allows one to generate image contrast that is not only related to the attenuation of the MR-invisible macromolecular protons but also to their biophysical and chemical environment. ${ }^{11}$

Received April 27, 2011; accepted after revision June 20

From the Departments of Neurology (S.R., R.S., C.E., N.P.M., F.F.) and Radiology (C.E.), Medical University of Graz, Graz, Austria; and JSW Life Sciences (M.W.), Grambach, Austria.

Please address correspondence to Stefan Ropele, PhD, Department of Neurology, Medical University of Graz, Auenbruggerplatz 22, A-8036 Graz, Austria; e-mail: stefan.ropele@ medunigraz.at

http://dx.doi.org/10.3174/ajnr.A2812
In $\mathrm{AD}$, the MTR, a semiquantitative measure of the magnetization transfer contrast, has therefore been used to search for tissue changes that may provide complementary information to brain atrophy. Reduced MTR values have been observed in the hippocampus ${ }^{12,13}$ and in the temporal lobes ${ }^{14}$ of AD patients. Results from volumetric MTR analyses have even suggested that MTR changes are not restricted to the temporal lobes but can be found more globally throughout the brain. ${ }^{15-17}$ They also seem to occur independent from atrophy, or may be seen before gross structural changes take place. ${ }^{12,14,17,18}$ Some researchers have also reported an association between regional or global MTR reductions and cognitive decline. ${ }^{12,15,19}$

From these findings it could be speculated that MTR measurements might be a sensitive tool for following the evolution of tissue damage in AD. However, MTI data have not yet been accumulated in a longitudinal manner with this indication. We therefore used a double-blind, placebo-controlled trial of memantine and a healthy control group to investigate the capability of MTI for monitoring the progression of tissue changes in AD. We also investigated the association of MTI findings over time with volume changes in distinct compartments of the brain and with cognitive performance.

\section{Materials and Methods}

\section{Subjects}

This investigation was performed as an exploratory substudy of a placebo-controlled treatment trial with memantine, which has been described in more detail elsewhere. ${ }^{20}$ Inclusion criteria for the trial were a diagnosis of probable $\mathrm{AD}$, as identified by NINCDS-ADRDA 
criteria, ${ }^{1}$ and fulfillment of the DSM-IV criteria $^{21}$ for dementia of the Alzheimer type. Twenty-eight of the 36 patients ( 14 women) in the trial volunteered to participate in the MTI substudy. Their mean age was $76.5(\mathrm{SD}=5.8)$ years and they had mild to moderate dementia, as defined by MMSE scores, ${ }^{22}$ which ranged from 14-24 (mean $18.9 \pm$ $2.7 \mathrm{SD}$ ). Half of the patients were assigned to receive memantine in a dose of $20 \mathrm{mg} /$ day, and half received placebo treatment. Baseline and follow-up examinations after 6 and 12 months consisted of a comprehensive clinical and imaging battery, ${ }^{20}$ and the MMSE scoring at each time point was done by a single rater, unaware of the imaging findings. Follow-up examinations at month 6 were performed in $27 \mathrm{AD}$ patients, and 18 participated in the follow-up examination at month 12.

A healthy control group consisting of 8 men and 11 women was used to obtain reference MTR values. Their mean age was 73.3 (SD = 3.2) years and they had a mean MMSE of 27.6 ( $\mathrm{SD}=0.8$ ). These individuals were assessed by a structured clinical interview, and a physical and neurologic examination, to assure that they were free of any overt neurologic or psychiatric disorder. Repeat cognitive assessment after 12 months in 17 of these volunteers revealed no change in the MMSE score (27.9 [1.3]); a follow-up MR imaging was not obtained.

The study protocol was approved by the local ethics committee and written informed consent was obtained from the patients and their caregivers.

\section{MR Imaging}

MR imaging was performed on a $1.5 \mathrm{~T}$ whole body scanner (Philips Intera; Philips Medical Systems, Best, the Netherlands) and included conventional imaging and MT imaging.

The MT sequence was based on a spoiled 2D gradient-echo sequence $\left(\mathrm{TR}=480 \mathrm{~ms} ; \mathrm{TE}=10 \mathrm{~ms}\right.$; flip angle $=50^{\circ}$; number of sections $=24 ;$ section thickness $=5 \mathrm{~mm}$; gap $=0.5 \mathrm{~mm} ; \mathrm{FOV}=250$ $\mathrm{mm}$; matrix $=128 \times 256$; number of averages $=1)$ that was performed with and without a binomial MT saturation pulse $\left(90^{\circ}-180^{\circ}-\right.$ $90^{\circ}$, maximum amplitude $=21 \mu \mathrm{T}$ ). To reduce the sensitivity for motion-induced artifacts, which can be an issue with demented patients, a $2 \mathrm{D}$, instead of a 3D, acquisition technique was used and the total measurement time was reduced to 2 minutes. The latter was achieved by using only 1 average, a reduced acquisition matrix, and a binomial saturation pulse with a duration of less than $1 \mathrm{~ms}$ instead of a much longer off-resonant saturation pulse.

The conventional protocol included an axial FLAIR sequence $(\mathrm{TR}=6000 \mathrm{~ms} ; \mathrm{TE}=130 \mathrm{~ms} ;$ inversion time $=1200 \mathrm{~ms} ; \mathrm{FOV}=230$ $\mathrm{mm}$; matrix $=256 \times 256$; section thickness $=5 \mathrm{~mm})$, an axial T2weighted FSE sequence $(\mathrm{TR}=3900 \mathrm{~ms}$; $\mathrm{TE}=80 \mathrm{~ms}$; FOV $=230 \mathrm{~mm}$; matrix $=256 \times 256$; section thickness $=5 \mathrm{~mm})$, and a volumetric MPRAGE sequence (flip angle $=15^{\circ} ; \mathrm{TR}=20 \mathrm{~ms}$; $\mathrm{TE}=4.5 \mathrm{~ms}$; $\mathrm{TI}=$ $400 \mathrm{~ms}$ ) with whole brain coverage. The MPRAGE sequence was acquired in the coronal plane with a $1.0-\times-1.0-\mathrm{mm}$ in-plane resolution and with 1.2-mm-thick partitions.

Follow-up scans were repositioned carefully using a line that joined the most inferior-anterior and inferior-posterior parts of the corpus callosum as reference.

\section{Image Analysis}

MTR maps were calculated according to the formula MTR = $\left(M_{s s}-M_{0}\right) / M_{0}$, where $M_{s s}$ and $M_{0}$ are the signal intensities obtained with and without MT saturation, respectively. MTR values were assessed globally by means of a histogram analysis. As the histogram peak mainly reflects white matter properties, we additionally measured the MTR in a regional manner. For this analysis we only considered those gray matter structures that could be assessed reliably within the constraints of image resolution of the MTR maps. The regional analysis therefore included the thalamus, the putamen, the caudate nucleus, and the hippocampus. This was performed by a single operator, who manually outlined these regions in the left and right hemisphere, and was unaware of all other clinical and imaging information.

For the histogram analysis, nonbrain tissue was removed in the MTR maps with the brain extraction tool BET, which is part of the University of Oxford's FMRIB software library (FSL; http://www.fmrib.ox.ac.uk/fsl). All remaining voxels were then considered for the MTR histogram analysis. For each histogram, the mean value, the peak position-that is, the MTR value with the highest frequencyand the relative peak height - that is, the relative voxel count at the peak position-were calculated using home-written programs. To correct for differences in individual brain volumes, the histograms were normalized by the total number of voxels contributing to the histogram.

In $\mathrm{AD}$ patients, whole brain atrophy rates were calculated from the MPRAGE scans with the fully automated structural image evaluation by using normalization of atrophy (SIENA) method, which is also part of FSL. Hippocampal volumes were assessed on the coronal MPRAGE sections by manual tracing using neuroanatomic atlases. ${ }^{20}$ Atrophy rates were calculated for the interval from baseline to month 6 and from baseline to month 12. In addition, normalized brain volumes were calculated at baseline using the SIENAX method from FSL. Performing regional analyses, normalized volumes were calculated for gray and white matter, for the cortex, and for the ventricles.

All image analyses were done without demographic and clinical information.

\section{Statistical Analysis}

We used the Statistica package for Windows (StatSoft, Tulsa, Oklahoma) for data analysis. Because of the exploratory nature of this investigation, with a relatively small patient number in treatment subgroups, we decided to perform all analyses on the combined cohort first. In a second step, we looked for possible differences in the direction and magnitude of changes between treatment subgroups. A Student $t$ test was used to assess differences in regional and global MTR between $\mathrm{AD}$ patients and controls. Longitudinal changes in MTR metrics and the MMSE of AD patients were tested with an ANOVA. For comparison with baseline MTR values, a Student $t$ test for paired samples was used. Univariate linear regression analysis was used to explore the association of the MTR and brain volume with cognition, and also to explore the effect of regional atrophy on histogram parameters. A $P$ value of less than .05 was considered to indicate statistical significance.

\section{Results}

At baseline, $\mathrm{AD}$ patients showed moderate but significant reductions in all histogram-derived global MTR metrics when compared with the healthy controls (Table 1). Regional MTR values of the hippocampal region were also significantly lower in $\mathrm{AD}$ patients than controls, whereas, at first assessment, no significant differences were seen in the putamen, caudate nucleus, and thalamus.

Longitudinal analysis of the serial MTR histograms of AD patients showed a constant and significant progression of 


\begin{tabular}{|c|c|c|c|c|}
\hline & Controls & AD Baseline & AD Month 6 & AD Month 12 \\
\hline$n$ & 19 & 28 & 27 & 18 \\
\hline $\operatorname{Sex}(M / F)$ & $8 / 11$ & $14 / 14$ & $14 / 13$ & $8 / 10$ \\
\hline Age & $73.3(3.2)$ & $76.5(5.8)$ & $76.5(5.9)$ & $76.1(5.8)$ \\
\hline \multicolumn{5}{|l|}{ Global MTR } \\
\hline Histogram peak position [\%] & $67.21(0.68)$ & $66.51(1.0)^{\mathrm{a}}$ & $65.77(0.90)^{\mathrm{b}}$ & $65.46(1.20)^{\mathrm{b}}$ \\
\hline Histogram peak height [a.u.] & $0.591(0.04)$ & $0.554(0.04)^{\mathrm{a}}$ & $0.515(0.07)^{\mathrm{b}}$ & $0.501(0.06)^{b}$ \\
\hline Mean histogram MTR [\%] & $55.26(1.30)$ & $53.55(1.50)^{\mathrm{a}}$ & $51.63(1.37)^{\mathrm{b}}$ & $50.89(1.45)^{\mathrm{b}}$ \\
\hline \multicolumn{5}{|l|}{ Regional MTR } \\
\hline Hippocampus MTR [\%] & $63.68(1.51)$ & $62.51(1.21)^{\mathrm{a}}$ & $61.04(1.42)^{\mathrm{b}}$ & $60.48(1.15)^{b}$ \\
\hline Caudate nucleus MTR [\%] & $57.51(1.01)$ & $57.15(1.24)$ & $55.52(2.23)^{\mathrm{b}}$ & $54.7(2.06)^{\mathrm{b}}$ \\
\hline Thalamus MTR [\%] & $62.51(1.47)$ & $63.33(1.52)$ & $61.78(1.99)^{\mathrm{b}}$ & $61.35(2.35)^{\mathrm{b}}$ \\
\hline Putamen MTR [\%] & $57.74(1.13)$ & $58.04(1.49)$ & $58.12(1.21)$ & $57.59(1.47)^{\mathrm{b}}$ \\
\hline Brain volume loss [\%] & NA & NA & 1.04 (1.68) & $2.22(1.87)$ \\
\hline Hippocampal volume loss [\%] & NA & NA & 1.77 (2.99) & $2.86(3.62)$ \\
\hline MMSE & $27.6(0.8)$ & $18.9(2.8)$ & $18.8(4.8)$ & $18.6(4.3)$ \\
\hline
\end{tabular}

Note:-For the regional MTR analysis, values from both hemispheres were averaged; a.u. indicates arbitrary unit(s).

a Significant difference between $A D$ patients and controls.

b Significant difference from baseline.

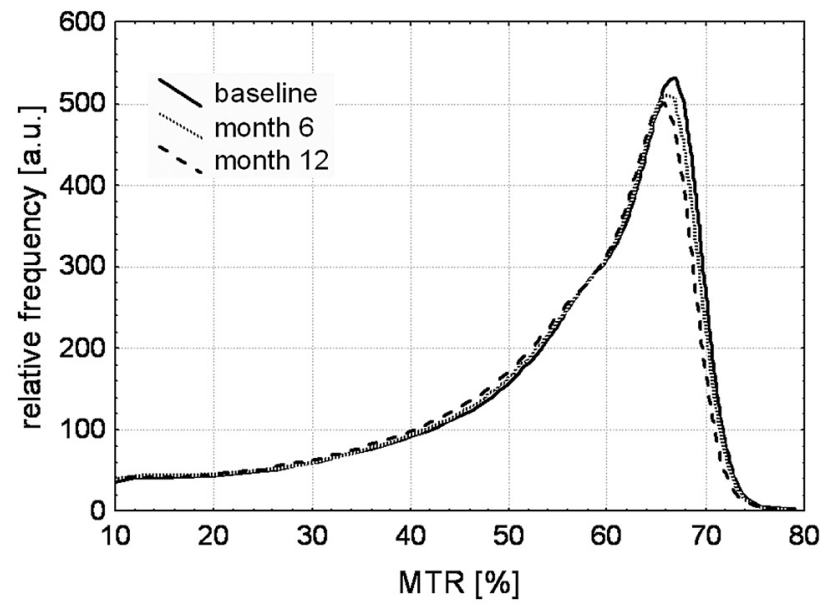

Fig 1. Averaged histograms from all $A D$ patients for baseline and follow-up scans. Already at month 6 , a reduction of both the peak position and the relative peak height are clearly visible.

brain tissue changes over the 1-year period (Fig 1). Significant changes in all histogram parameters - that is, mean MTR, peak position, and relative peak height-were seen already after 6 months, with the peak height and the mean MTR showing the most pronounced changes (Table 1). These global changes were paralleled by regional MTR decreases in assessed structures such as the hippocampus and also in the putamen and thalamus. In the caudate nucleus, a significant decrease of the MTR was seen only after 12 months. When performing the longitudinal analysis in the subgroups of patients treated with memantine or placebo, we found similar trends as for the entire cohort in both subgroups, but all differences became nonsignificant. There was a constant loss of whole-brain volume over the study period, which was in a magnitude of $2.2 \%$ per year. The average loss of the hippocampal volume after 1 year was $2.9 \%$. Cognition also constantly deteriorated over time, but the difference between baseline and 12-month follow-up was not significant (Table 1).

When looking for an association between MTR histogram metrics and whole brain atrophy, we noted a strong correlation of the peak height with the normalized brain volume $(r=$ $0.63, P<.01)$. This association was mediated by the white
Table 2: Relation between deep gray matter MTR and cognition in $\mathrm{AD}$ patients at baseline

\begin{tabular}{lcc}
\hline & \multicolumn{2}{c}{ MMSE } \\
\cline { 2 - 3 } & $r$ & $p$ \\
\hline Left hippocampus & 0.57 & $<0.01$ \\
Right hippocampus & 0.38 & 0.048 \\
Left putamen & 0.70 & $<0.001$ \\
Right putamen & 0.60 & $<0.01$ \\
Left thalamus & 0.52 & $<0.01$ \\
Right thalamus & 0.42 & 0.029 \\
Left caudate nucleus & 0.07 & 0.69 \\
Right caudate nucleus & -0.06 & 0.72 \\
\hline
\end{tabular}

Note:-Stronger correlations can be consistently observed in the left hemisphere.

matter volume only $(r=0.68, P<.01)$. No associations were seen between peak position and normalized volumes of gray and white matter, or with the ventricular volume.

We found no correlation between the MTR histogram parameters at baseline and their change over time with the respective MMSE scores of AD patients. There was also no association between brain volume measurements, including normalized brain volume, cortical volume, ventricular volume, gray and white matter volumes, and the MMSE. In contrast, regional MTR values in the thalamus, putamen, and hippocampus were highly correlated with the MMSE in AD patients (Table 2). While no interhemispheric MTR differences in basal structures were found, MTR values in the left hemisphere showed a substantially better correlation with cognition compared with the right hemisphere. A representative example for this hemispheric difference at all time points is given in Fig 2 in relation to the hippocampus.

\section{Discussion}

In this first longitudinal MTR study of patients with mild to moderate $\mathrm{AD}$, we confirmed the presence of widespread changes throughout the brain of Alzheimer patients and observed a substantial decline of both global and regional MTR values over a period of 1 year. Despite the relatively small cohort, changes over time were already significant after 6 months and most pronounced in those regions that already showed a significant MTR reduction at baseline when com- 

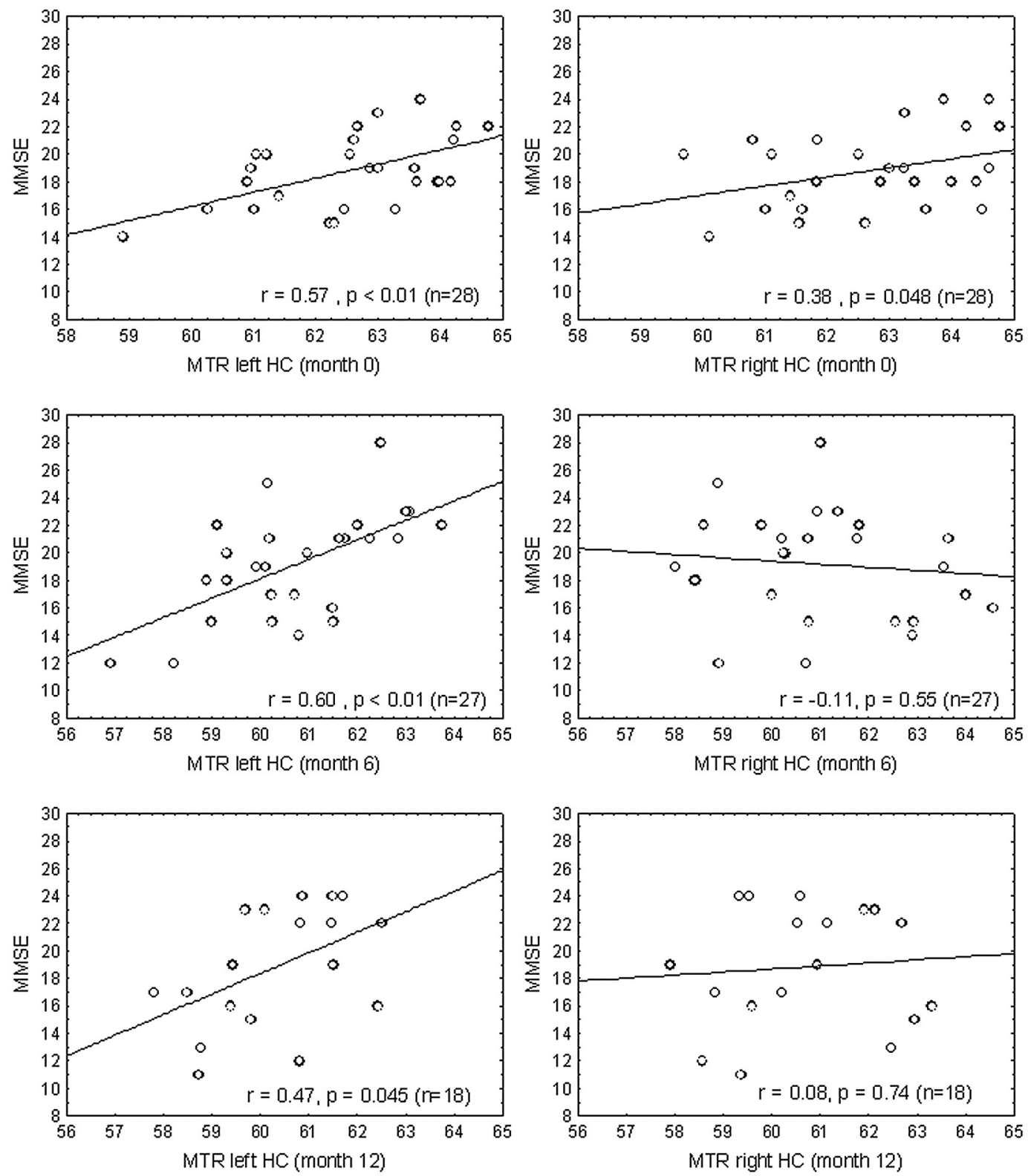

Fig 2. Hemispheric difference of the association between MTR and MMSE in the hippocampus (HC) with a constantly stronger relationship on the left hemisphere.

pared with healthy controls. MTR changes were noted irrespective of treatment, which might suggest that memantine has no effect on the microstructure of the brain tissue. However, this study was neither planned nor powered to assess treatment effects with MTI.

When interpreting these results, it has to be considered that MTR reductions were also accompanied by a substantial loss of brain volume of approximately $2.2 \%$ in 1 year, which corresponds well with previous reports. ${ }^{23,24}$ It could therefore be speculated that tissue loss per se could have caused most of the reductions seen in the MTR histogram metrics. In fact, according to theoretic considerations, and as also revealed by linear regression analysis in this study, a reduction of the white matter volume reduces the peak height of the MTR histogram. White matter atrophy, however, has no effect on the peak position. This is attributable to the fact that the histogram peak position indicates the MTR of the most abundant tissue com- partment, which, in the brain, is white matter. Consequently, the peak position represents the mean MTR of white matter and the peak height reflects its relative volume. Thus, a change in peak position is indicative of a change in the composition of the largest portion of white matter and not a consequence of volume loss. The results of the MTR analysis of the deep gray matter structures-which also showed a constant decrease of the MTR and were assessed by a region of interest analysis, which is not susceptible to tissue loss-support this assumption.

Following reports of widespread tissue damage in $\mathrm{AD},{ }^{15-17}$ we attempted to assess global tissue changes by means of a fully automated and robust histogram analysis, which is independent of operator bias. As the histogram provides less information on gray matter structures, we additionally performed ROI analyses in the hippocampus and the deep gray matter structures. Only recently, the latter have been identified as regions 
with a high susceptibility for AD pathology. ${ }^{25}$ Our findings of a progressive MTR reduction in the basal ganglia and thalamus are in line with the observation of a significant atrophy of the putamen and thalamus in $\mathrm{AD} .{ }^{25}$ We would also have expected to find MTR reductions in the cortex. ${ }^{16,26}$ However, due to limited image resolution and partial volume effects, we unfortunately were not able to obtain reliable and reproducible MTR values in the cortex, and therefore abstained from such analysis.

The MTR values obtained in this work are substantially higher than those published so far and are the result of our efforts to maximize the MT contrast. This was mainly achieved by using ultra-short ( $1 \mathrm{~ms}$ ) binomial MT saturation pulses instead of long-lasting off-resonant saturation pulses and also by a short interpulse delay, that is, the saturation pulse was played out every $20 \mathrm{~ms}$. Overall, this setting allowed for a higher saturation effect while keeping the SAR moderate. Binomial saturation pulses are more susceptible for direct saturation effects, which may have additionally contributed to the high MTR values. ${ }^{27}$ In contrast to off-resonant saturation pulses, binomial pulses are T2-selective, which means that they do not only saturate macromolecular protons but are also sensitive to T2 changes of the free mobile tissue water. An increase of T2, a condition that has been observed in $\mathrm{AD},{ }^{28,29}$ would therefore also cause a reduction of the MTR. We thus cannot exclude that this effect may have impacted our results. Recent work has demonstrated that binomial saturation is sensitive enough to assess age-related tissue changes in normal subjects and to identify risk factors for microstructural tissue damage. ${ }^{30,31}$ Here, the multisection acquisition mode, the reduced acquisition matrix, and, consequently, the reduction of the acquisition time allowed for the reduction of motion-induced artifacts while still providing a high signal intensity-tonoise ratio and a strong MT contrast.

While the observation of a constant MTR reduction suggests MTI as a potent new technique for following the progression of $\mathrm{AD}$, there may be a concern that observed changes have been a consequence of technical instability, for example, a scanner drift. A strong argument against this is the fact that reference, baseline, and follow-up scans of different patients were acquired in an interleaved fashion over a period of 30 months. Furthermore, the relative magnitude of MTR reduction was not constant in the regions assessed. The observed MTR decrease therefore most likely reflects progression of microstructural tissue damage associated with AD pathology. In this context, it should also be considered that a major scannerrelated source of MTR variations are coil- and head-size-specific inhomogeneities of the radio frequency transmit field. ${ }^{32}$ This means that intrasubject MTR variations are likely to be much smaller than intersubject variations if the patient is properly repositioned, such as in our study. In this context, it appears important to note that the coefficients of variation for intrasubject variation of the histogram metrics in the control group were clearly below the relative change of these variables over the 12-month period in the $\mathrm{AD}$ cohort.

Performed associations of the MTR with the degree of cognitive dysfunction, as measured by the MMSE, have several limitations. Previous reports demonstrated an association between histogram parameters and cognition, ${ }^{15,19}$ whereas others have not. ${ }^{17,33}$ The histogram parameters analyzed here also did not show any association with cognition. One possible explanation is that we performed our analyses on a relatively small cohort of patients with mild to moderate $\mathrm{AD}$, while van Flier et $\mathrm{al}^{15}$ performed an analysis across different groups of dementia severity, including subjects with mild cognitive impairment and thus a much larger range of cognitive performance. Another explanation might come from the fact that we only used the MMSE as a measure of cognitive impairment, which certainly does not reflect the full range of cognitive deficits. On the other hand, and in line with previous studies including attempts to quantify fundamental MT parameters such as the molar fraction of myelin bound protons, ${ }^{12,34}$ we found a strong association between the MMSE score and the hippocampal MTR. This could also suggest that AD-related brain damage, as depicted by MTR, may escape clinical detection unless it occurs in clinically eloquent regions or in other words that more diffuse changes in $\mathrm{AD}$ brains as depicted by MTR histogram analysis might progress without overt clinical signs. We also found no correlation of the change in the MTR of specific brain regions with cognitive performance over time, but this analysis was possibly flawed by the fact that no overall change in the MMSE of our patients occurred over the 1 -year period.

Interestingly, the associations between the hippocampal MTR and the MMSE were stronger for the left hippocampus, and the same laterality effect was also seen for the putamen and thalamus. The origin of this asymmetry, which has also been observed in volumetric analyses, ${ }^{25}$ has not been resolved so far, but it is quite plausible that structural damage of the left hemisphere is more relevant for the cognitive functions assessed. Thus, our MTR findings parallel the observation that volume loss of the left putamen and thalamus is a strong predictor for cognitive decline in $\mathrm{AD}$, while the volume of the caudate nucleus was not associated with cognition. ${ }^{25}$

\section{Conclusions}

This study extends previous reports on the utility of MTI for assessing structural tissue damage in $\mathrm{AD}$. The results indicate a very high sensitivity of MTR histogram metrics for the progression of global brain changes in $\mathrm{AD}$, as we observed a significant decline of all MTR histogram metrics within 6 months. The fact that a parallel decrease of the MTR in the hippocampus and in other deep gray matter structures can also be observed by regional analyses supports the use of MTI as a marker for disease progression in AD. Such measurements may be more sensitive to dynamic changes than the clinical assessment.

Disclosures: As indicated in the Introduction and Methods, submitted work was performed as an add-on to a pilot study with memantine, which was supported by Merz Pharma Austria GmbH (Schmidt R. et al, JNNP2008;79:1312-17). The data acquisition and analyses needed for present study, however, were solely driven by academic interest and performed as part of academic research without any involvement or support of Merz Pharma Austria GmbH or any other sponsor. Reinhold Schmidt-UNRELATED: Grants/Grants Pending Era-Net Neuron Austrian Science Fund; Payment for Lectures, Including Service on Speakers Bureaus: Pfizer, Merz Austria, Novartis. Manfred Windisch_UNRELATED: Employment: JSW-Lifesciences (CEO).

\section{References}

1. McKhann G, Drachman D, Folstein M, et al. Clinical diagnosis of Alzheimer's disease: report of the NINCDS-ADRDA Work Group under the auspices of 
Department of Health and Human Services Task Force on Alzheimer's Disease. Neurology 1984;34:939-44

2. Braak H, Braak E. Neuropathological staging of Alzheimer-related changes. Acta Neuropathol 1991;82:239-59

3. Hyman BT, Van Hoesen GW, Damasio AR, et al. Alzheimer's disease: cellspecific pathology isolates the hippocampal formation. Science $1984 ; 225: 1168-70$

4. Arnold SE, Hyman BT, Flory J, et al. The topographical and neuroanatomical distribution of neurofibrillary tangles and neuritic plaques in the cerebral cortex of patients with Alzheimer's disease. Cereb Cortex 1991;1:103-16

5. Mitew S, Kirkcaldie MT, Halliday GM, et al. Focal demyelination in Alzheimer's disease and transgenic mouse models. Acta Neuropathol 2010;119:567-77

6. Ihara M, Polvikoski TM, Hall R, et al. Quantification of myelin loss in frontal lobe white matter in vascular dementia, Alzheimer's disease, and dementia with Lewy bodies. Acta Neuropathol 2010;119:579-89

7. Sluimer JD, van der Flier WM, Karas GB, et al. Whole-brain atrophy rate and cognitive decline: longitudinal MR study of memory clinic patients. Radiology 2008;248:590-98

8. Henneman WJ, Sluimer JD, Barnes J, et al. Hippocampal atrophy rates in Alzheimer disease: added value over whole brain volume measures. Neurology 2009;72:999-1007

9. Balaban RS, Ceckler TL. Magnetization transfer contrast in magnetic resonance imaging. Magn Reson Q 1992;8:116-37

10. Schmierer K, Scaravilli F, Altmann DR, et al. Magnetization transfer ratio and myelin in postmortem multiple sclerosis brain. Ann Neurol 2004;56:407-15

11. Wolff SD, Balaban RS. Magnetization transfer imaging: practical aspects and clinical applications. Radiology 1994;192:593-99

12. Hanyu H, Asano T, Iwamoto T, et al. Magnetization transfer measurements of the hippocampus in patients with Alzheimer's disease, vascular dementia, and other types of dementia. AJNR Am J Neuroradiol 2000;21:1235-42

13. Hanyu H, Asano T, Sakurai H, et al. Magnetization transfer measurements of the hippocampus in the early diagnosis of Alzheimer's disease. J Neurol Sci 2001;188:79-84

14. Kabani NJ, Sled JG, Shuper A, et al. Regional magnetization transfer ratio changes in mild cognitive impairment. Magn Reson Med 2002;47:143-48

15. van der Flier WM, van den Heuvel DM, Weverling-Rijnsburger AW, et al. Magnetization transfer imaging in normal aging, mild cognitive impairment, and Alzheimer's disease. Ann Neurol 2002;52:62-67

16. Bozzali M, Franceschi M, Falini A, et al. Quantification of tissue damage in AD using diffusion tensor and magnetization transfer MRI. Neurology 2001;57:1135-37

17. Ridha BH, Symms MR, Tozer DJ, et al. Magnetization transfer ratio in Alzheimer disease: comparison with volumetric measurements. AJNR Am I Neuroradiol 2007;28:965-70

18. Kabani NJ, Sled JG, Chertkow H. Magnetization transfer ratio in mild cogni- tive impairment and dementia of Alzheimer's type. Neuroimage 2002;15: 604-10

19. Van Der Flier WM, Van Den Heuvel DM, Weverling-Rijnsburger AW, et al. Cognitive decline in $\mathrm{AD}$ and mild cognitive impairment is associated with global brain damage. Neurology 2002;59:874-79

20. Schmidt R, Ropele S, Pendl B, et al. Longitudinal multimodal imaging in mild to moderate Alzheimer disease: a pilot study with memantine. J Neurol Neurosurg Psychiatry 2008;79:1312-17

21. American Psychiatric Association. Diagnostic and Statistical Manual of Mental Disorders DSM-IV. Washington, DC: Author, 1994

22. Folstein MF, Folstein SE, McHugh PR. "Mini-mental state." A practical method for grading the cognitive state of patients for the clinician. J Psychiatr Res 1975;12:189-98

23. Chan D, Fox NC, Jenkins R, et al. Rates of global and regional cerebral atrophy in AD and frontotemporal dementia. Neurology 2001;57:1756-63

24. Sluimer JD, Vrenken $\mathrm{H}$, Blankenstein MA, et al. Whole-brain atrophy rate in Alzheimer disease: identifying fast progressors. Neurology 2008;70:1836-41

25. de Jong LW, van der Hiele K, Veer IM, et al. Strongly reduced volumes of putamen and thalamus in Alzheimer's disease: an MRI study. Brain 2008;131:3277-85

26. Ginestroni A, Battaglini M, Della Nave R, et al. Early structural changes in individuals at risk of familial Alzheimer's disease: a volumetry and magnetization transfer MR imaging study. J Neurol 2009;256:925-32

27. Hua J, Hurst GC. Analysis of on- and off-resonance magnetization transfer techniques. J Magn Reson Imaging 1995;5:113-20

28. Kirsch SJ, Jacobs RW, Butcher LL, et al. Prolongation of magnetic resonance T2 time in hippocampus of human patients marks the presence and severity of Alzheimer's disease. Neurosci Lett 1992;134:187-90

29. Wang H, Yuan H, Shu L, et al. Prolongation of T(2) relaxation times of hippocampus and amygdala in Alzheimer's disease. Neurosci Lett 2004;363: $150-53$

30. Fazekas F, Ropele S, Enzinger C, et al. MTI of white matter hyperintensities. Brain 2005;128:2926-32

31. Ropele S, Enzinger C, Sollinger M, et al. The impact of sex and vascular risk factors on brain tissue changes with aging: magnetization transfer imaging results of the Austrian stroke prevention study. AJNR Am J Neuroradiol 2010;31:1297-301

32. Ropele S, Filippi M, Valsasina P, et al. Assessment and correction of B1-in duced errors in magnetization transfer ratio measurements. Magn Reson Med 2005;53:134-40

33. van Es AC, van der Flier WM, Admiraal-Behloul F, et al. Lobar distribution of changes in gray matter and white matter in memory clinic patients: detected using magnetization transfer imaging. AJNR Am J Neuroradiol 2007;28: $1938-42$

34. Ridha BH, Tozer DJ, Symms MR, et al. Quantitative magnetization transfer imaging in Alzheimer disease. Radiology 2007;244:832-37 